\title{
NECESSARY CONDITIONS FOR STABILITY OF DIFFEOMORPHISMS
}

\author{
BY \\ JOHN FRANKS $\left({ }^{1}\right)$
}

\begin{abstract}
S. Smale has recently given sufficient conditions for a diffeomorphism to be $\Omega$-stable and conjectured the converse of his theorem. The purpose of this paper is to give some limited results in the direction of that converse. We prove that an $\Omega$ stable diffeomorphism $f$ has only hyperbolic periodic points and moreover that if $p$ is a periodic point of period $k$ then the $k$ th roots of the eigenvalues of $d f_{p}^{k}$ are bounded away from the unit circle. Other results concern the necessity of transversal intersection of stable and unstable manifolds for an $\Omega$-stable diffeomorphism.
\end{abstract}

Introduction. We will say that a diffeomorphism $f: M \rightarrow M$ of a compact manifold is $\Omega$-stable if (a) there is a neighborhood $N(f)$ of $f$ in the $C^{1}$ topology such that $g \in N(f)$ implies there is a homeomorphism $h$ from the nonwandering set of $f$, $\Omega(f)$ to the nonwandering set of $g, \Omega(g)$ which satisfies $g \cdot h=h \cdot f$; and (b) if $p$ is a periodic point of $f$ then $\operatorname{dim} W^{s}(p ; f)=\operatorname{dim} W^{s}(h(p) ; g)$. Property $(b)$ is not usually included in the definition of $\Omega$-stable (see [3]), but it is a weak condition which is very natural and is apparently necessary for the proof of one of our lemmas (2.2). In his paper [4], S. Smale provides sufficient conditions for a diffeomorphism to be $\Omega$-stable. One of his conditions is that the nonwandering set have a hyperbolic structure. Recall that a closed invariant set $\Lambda$ is said to have a hyperbolic structure if

(a) There is continuous splitting of the restriction of the tangent bundle to $\Lambda$, $T M_{\Lambda}=E^{s} \oplus E^{u}$ which is preserved by the derivative $d f$.

(b) There exist constants $C>0, C^{\prime}>0$ and $\lambda \in(0,1)$ and a Riemannian metric \| $\|$ on $T M_{\Lambda}$ such that

$$
\left\|d f^{n}(v)\right\|<C \lambda^{n}\|v\| \quad \text { for } v \in E^{s} \text { and } n>0,
$$

and

$$
\left\|d f^{n}(v)\right\| \geqq C^{\prime} \lambda^{-n}\|v\| \quad \text { for } v \in E^{u} \text { and } n>0 .
$$

One would like to prove that the condition above is necessary for $\Omega$-stability. In this paper we give results which are a start in that direction. I would like to thank

Presented to the Society, January 24, 1971; received by the editors February 18, 1970 and, in revised form, October 27, 1970.

AMS 1970 subject classifications. Primary 58F10, 58F15.

Key words and phrases. $\Omega$-stability, structural stability, hyperbolic structure, nonwandering set.

(1) Research sponsored by the Air Force Office of Scientific Research, Office of Aerospace Research under Contract No. F44620-67-C-0029.

Copyright (C) 1971, American Mathematical Society 
M. Hirsch and M. Shub for valuable conversations concerning Theorem 1 . Throughout we will consider only $C^{\infty}$ compact manifolds and $C^{\infty}$ diffeomorphisms.

THEOREM 1. If $f: M \rightarrow M$ is $\Omega$-stable then all the periodic points of $f$ are hyperbolic and there exists a constant $\lambda \in(0,1)$ such that for any periodic point $p$ the inequalities

$$
\begin{array}{ll}
\left\|d f^{n}(v)\right\| \leqq C_{p} \lambda^{n}\|v\| \quad \text { for } v \in E_{p}^{s} \text { and } n>0, \\
\left\|d f^{n}(v)\right\| \geqq C_{p}^{-1} \lambda^{-n}\|v\| \quad \text { for } v \in E_{p}^{u} \text { and } n>0
\end{array}
$$

are satisfied, where $C_{p}$ is a positive constant depending on $p$.

We remark that a diffeomorphism needs to satisfy only part (a) of the definition of $\Omega$-stability in order to satisfy the conclusion of this theorem. Part (b) of the definition is not used in the proof.

M. Shub pointed out to me that if one could obtain the above result with a single constant $C$ independent of $p$ then the necessity of a hyperbolic structure on the nonwandering set would follow.

We also investigate how the stable and unstable manifolds of an $\Omega$-stable diffeomorphism fit together. Following Abraham and Smale [1] we define a subbasic set for $f \in \operatorname{Diff}(M)$ to be a compact invariant set $\Lambda \subset M$ with hyperbolic structure such that $\left.f\right|_{\Lambda}$ is topologically transitive and the periodic points are dense in $\Lambda$.

Definition. Two subbasic sets $\Lambda_{1}$ and $\Lambda_{2}$ are attached if there are periodic points $p_{1}, q_{1} \in \Lambda_{1}$ and $p_{2}, q_{2} \in \Lambda_{2}$ such that $W^{s}\left(p_{1}\right) \cap W^{u}\left(p_{2}\right) \neq \varnothing$ and $W^{u}\left(q_{1}\right) \cap$ $W^{s}\left(q_{2}\right) \neq \varnothing$.

THEOREM 2. If $f: M \rightarrow M$ is $\Omega$-stable and $\Lambda_{1}$ and $\Lambda_{2}$ are subbasic sets which are attached, then $\operatorname{dim} E^{s}\left(\Lambda_{1}\right)=\operatorname{dim} E^{s}\left(\Lambda_{2}\right)$. If $x \in \Lambda_{1}$ and $y \in \Lambda_{2}$ then $W^{s}(x)$ and $W^{u}(y)$ always intersect transversely and moreover there is a constant $\alpha>0$ such that for any $x, y$ and any point of intersection of $W^{s}(x)$ and $W^{u}(y)$ the angle between $W^{s}(x)$ and $W^{u}(y)$ is greater than $\alpha$.

By the angle between two subspaces $V_{1}, V_{2}$ in $T M_{x}$ we of course mean $\inf \left(\operatorname{Cos}^{-1}\left(v_{1}, v_{2}\right)_{x}\right)$ where $v_{1} \in V_{1}, v_{2} \in V_{2}$ are unit vectors and $(,)_{x}$ is the inner product on $T M_{x}$.

1. The periodic points of $\Omega$-stable diffeomorphisms. We begin with a lemma which allows us to alter a diffeomorphism to achieve a desired derivative at a finite number of points.

(1.1) LemMA. Let $\theta$ be a finite set of points in $M$, let $Q=\bigoplus_{x \in \theta} T M_{x}$ and let $Q^{\prime}=\oplus_{x \in \theta} T M_{f(x)}$. If $\varepsilon>0$ is sufficiently small and $G: Q \rightarrow Q^{\prime}$ is an isomorphism such that $\|G-d f\|<\varepsilon / 10$ then there exists a diffeomorphism $g: M \rightarrow M, \varepsilon$ close to $f$ in the $C^{1}$ topology, such that $d g_{x}=G \mid T M_{x}$ for any $x \in \theta$. Moreover if $R$ is a compact subset of $M$ disjoint from $\theta$ we can require $f(x)=g(x)$ for $x \in R$. 
Proof. We assume for simplicity that the Riemannian metric on $M$ comes from an embedding $M \rightarrow R^{m}$ and the usual metric on $R^{m}$. Let exp be the restriction to $Q \cup Q^{\prime}$ of the exponential map from $T M$ to $M$ determined by the metric.

We now choose a number $\delta>0$ satisfying the following conditions:

(1) If $\theta \cup f(\theta)=\left\{x_{i}\right\}_{i=1}^{n}$ and $\hat{B}_{i}=\left\{v \mid v \in T M_{x_{i}}\right.$ and $\left.\|v\| \leqq \delta\right\}$ then exp: $\hat{B}_{i} \rightarrow M$ is an embedding, and if $B_{i}=\exp \left(\widehat{B}_{i}\right)$ then $B_{i}$ and $B_{j}$ are disjoint when $i \neq j$. We also make $\delta$ so small that, for all $i, R$ is disjoint from $B_{i}$.

(2) $\|\exp (v)-v\|<\varepsilon / 10$ if $v \in \hat{B}_{i}$ (recall \|\| is the norm on $R^{m}$ in which $M$ is embedded).

(3) $\left\|d \exp _{v}\right\|<1+\varepsilon / 10$ if $v \in \hat{B}_{i}$ and $\left\|d \exp _{x}^{-1}\right\|<1+\varepsilon / 10$ if $x \in B_{i}$.

(4) $\hat{f}: \hat{B}_{i} \rightarrow Q^{\prime}$ can be defined by $\hat{f}(v)=\exp ^{-1}(f(\exp (v)))$ and $\|G(u)-\hat{f}(u)\|<$ $(\varepsilon / 10)\|u\|$ and $\left\|G(v)-d \hat{f}_{u}(v)\right\|<(\varepsilon / 10)\|v\|$ hold when $u \in \hat{B}_{i}$ and $\hat{B}_{i} \subset Q$. (This is possible because $d f_{x_{i}}=d \hat{f}_{x_{i}}$ and $\left\|G-d f_{x_{i}}\right\|<\varepsilon / 10$.)

(5) If $K=\sup _{x \in M}\left\|d f_{x}\right\|$ then $\left\|d \exp _{u}-d \exp _{v}\right\|<\varepsilon / 10 K$ if $u, v \in \hat{B}_{i}$.

Choose a $C^{\infty}$ real-valued function $\sigma$ such that $0 \leqq \sigma(x) \leqq 1, \sigma(x)=0$ if $|x| \geqq \delta$, $\sigma(x)=1$ if $|x| \leqq \delta / 4$, and $0 \leqq \sigma^{\prime}(x)<2 / \delta$ for all $x$. Let $\rho: T M \rightarrow R$ be defined by $\rho(v)=\sigma(\|v\|)$.

We define the function $\hat{g}: \bigcup_{x_{i} \in \theta} \hat{B}_{i} \rightarrow T M$ by $\hat{g}(v)=\rho(v) G(v)+(1-\rho(v)) \hat{f}(v)$ and define $g: M \rightarrow M$ by $g(x)=\exp \left(\hat{g}\left(\exp ^{-1}(x)\right)\right)$ if $x \in \bigcup_{x_{i} \in \theta} B_{i}$ and $g(x)=f(x)$ otherwise. We will show that $g$ is $\varepsilon$ close to $f$ in the $C^{1}$ topology.

If $x \notin \bigcup_{x_{i} \in \theta} B_{i}, f(x)=g(x)$ and if $x \in \bigcup_{x_{i} \in \theta} B_{i}$,

$$
\begin{aligned}
\|f(x)-g(x)\| & =\left\|\exp \cdot \hat{f} \cdot \exp ^{-1}(x)-\exp \cdot \hat{g} \cdot \exp ^{-1}(x)\right\| \\
& \leqq \varepsilon / 5+\left\|\hat{f} \cdot \exp ^{-1}(x)-\hat{g} \cdot \exp ^{-1}(x)\right\|
\end{aligned}
$$

by condition (2) above. So if $v=\exp ^{-1}(x)$,

$$
\begin{aligned}
\|f(x)-g(x)\| & \leqq \varepsilon / 5+\|\hat{f}(v)-\rho(v) G(v)-(1-\rho(v)) \hat{f}(v)\| \\
& \leqq \varepsilon / 5+\rho(v)\|\hat{f}(v)-G(v)\| \\
& \leqq \varepsilon / 5+\varepsilon / 10<\varepsilon \quad \text { by (4) above. }
\end{aligned}
$$

We now check the difference in derivatives. If $v \in \bigcup_{x_{i} \in \theta} \hat{B}_{i}$,

So

$$
d \hat{g}_{v}(u)=\rho(v) G(u)+d \rho_{v}(u) G(v)+(1-\rho(v)) d \hat{f}_{v}(u)-d \rho_{v}(u) \hat{f}(v) .
$$

$$
\begin{aligned}
\left\|d \hat{f}_{v}(u)-d \hat{g}_{v}(u)\right\| & =\left\|\rho(v) G(u)-\rho(v) d \hat{f}_{v}(u)+d \rho_{v}(u)(G(v)-\hat{f}(v))\right\| \\
& \leqq \rho(v)\left\|G(u)-d \hat{f}_{v}(u)\right\|+\left\|d \rho_{v}(u)(G(v)-\hat{f}(v))\right\| .
\end{aligned}
$$

If $\|v\|>\delta, \rho(v)=0$; if $\|v\| \leqq \delta$,

$$
\left\|G(u)-d \hat{f}_{v}(u)\right\|<(\varepsilon / 10)\|u\| \quad \text { by (4) above. }
$$

If $\|v\| \geqq \delta d \rho_{v}(u)=0$; if $\|v\|<\delta\left\|d \rho_{v}\right\|<2 / \delta$ and $\|G(v)-\hat{f}(v)\|<(\varepsilon / 10)\|v\|<\varepsilon \delta / 10$, so

$$
\left\|d \rho_{v}(u)(G(v)-\hat{f}(v))\right\|<\frac{2}{\delta} \frac{\varepsilon \delta}{10}\|u\|=\frac{\varepsilon}{5}\|u\|
$$


Hence

$$
\left\|d \hat{f}_{v}(u)-d \hat{g}_{v}(u)\right\| \leqq \frac{\varepsilon}{10}\|u\|+\frac{\varepsilon}{5}\|u\|=\frac{3 \varepsilon}{10}\|u\|
$$

Let $y=\exp ^{-1}(x), z=\hat{f}(y)$, and $w=\hat{g}(y)$. Now

$$
\begin{aligned}
\left\|d f_{x}(v)-d g_{x}(v)\right\|= & \left\|d \exp _{z} \cdot d \hat{f}_{y} \cdot d \exp _{x}^{-1}(v)-d \exp _{w} \cdot d \hat{g} \cdot d \exp _{x}^{-1}(v)\right\| \\
\leqq & \left\|d \exp _{z} \cdot d \hat{f}_{y} \cdot d \exp _{x}^{-1}(v)-d \exp _{w} \cdot d \hat{f}_{y} \cdot d \exp _{x}^{-1}(v)\right\| \\
& +\left\|d \exp _{w} \cdot d \hat{f}_{y} \cdot d \exp _{x}^{-1}(v)-d \exp _{w} \cdot d \hat{g}_{y} \cdot d \exp _{x}^{-1}(v)\right\| \\
\leqq & \frac{\varepsilon}{10 K} \cdot K\left\|d \exp _{x}^{-1}(v)\right\|+\left(1+\frac{\varepsilon}{10}\right) \frac{3 \varepsilon}{10}\left\|d \exp _{x}^{-1}(v)\right\|
\end{aligned}
$$

by (3) and (5) above.

So

$$
\left\|d f_{x}(v)-d g_{x}(v)\right\| \leqq\left[\frac{\varepsilon}{10}+\left(1+\frac{\varepsilon}{10}\right) \frac{3 \varepsilon}{10}\right]\left(1+\frac{\varepsilon}{10}\right)\|v\|<\varepsilon\|v\| .
$$

Proof of Theorem 1. We first choose $\varepsilon>0$ sufficiently small that if $g$ is $\varepsilon$ close to $f$ in the $C^{1}$ topology then $f$ and $g$ are $\Omega$-conjugate.

For each periodic point $p$ of period $k$ we let $E_{p}^{s}$ be the sum of the eigenspaces of $d f_{p}^{k}$ in $T M_{p}$ which correspond to eigenvalues of absolute value less than or equal to one. If $V_{p}=\bigoplus_{i=1}^{k} E_{f^{i}(p)}^{s}$, then $V_{p}$ is a vector space of dimension $k\left(\operatorname{dim} E_{p}^{s}\right)$ and we will consider the isomorphism $L_{p}=d f \mid V_{p}$.

By the definition of $E_{p}^{s}$, the eigenvalues of $L_{p}$ must all have absolute value less than or equal to one. If there is a $\lambda \in(0,1)$ which is an upper bound for the set of all eigenvalues of all the isomorphisms $L_{p}$ (varying $p$ ), then it is clear that for each $p$ one can choose $C_{p}$ such that $\left\|d f^{n}(v)\right\|<C_{p} \lambda^{n}\|v\|$ for $v \in E_{p}^{s}$.

On the other hand if there is no such $\lambda$ then we can contradict the $\Omega$-stability of $f$ as follows: Choose a periodic point $p$ with an eigenvalue $\gamma$ such that $\left(|\gamma|^{-1}-1\right)<$ $\varepsilon / 20$. Let $Q_{p}=\oplus_{i=1}^{k} T M_{f^{i}(p)}$ where $k=$ period of $p$, and define $F: Q_{p} \rightarrow Q_{p}$ by $F(v)=|\gamma|^{-1} d f(v)$. $F$ has an eigenvalue of absolute value one, namely $\gamma /|\gamma|$. If $\gamma /|\gamma|$ is not a root of unity choose $G: Q_{p} \in Q_{p}$ such that $\|G-F\|<\varepsilon / 20$ and $G$ has an eigenvalue which is a root of unity; otherwise let $G=F$.

It follows that

$$
\|G-d f\| \leqq\|G-F\|+\|F-d f\|<\varepsilon / 10 .
$$

By (1.1) there exists a diffeomorphism $g: M \rightarrow M$ which is an $\varepsilon$ approximation to $f$ and with $d g_{p}=G \mid T M_{p}$.

$G$ has an eigenvalue which is a root of unity so there exists an $n$ such that $G^{n}$ has 1 as an eigenvalue. Recall that $k=$ the period of $p$. So there exists $v \in T M_{p}$ such that $\|v\|=1$ and $G^{n k}(v)=v$.

Let $\Delta=\max _{0 \leqq i \leqq n k}\left\|G^{i}\right\|$ and let $I=\{t v|0 \leqq| t \mid \leqq(\delta / 4) \Delta\}$ where $\delta$ is the $\delta$ of (1.1). Then for any vector $u \in I,\left\|G^{i}(u)\right\|<\delta / 4$ for any $i$. Referring back to the constructions 
in (1.1) we see if $u \in I$ then $\hat{g}^{n k}(u)=G^{n k}(u)=u$. That is, every point of $\exp (I)$ is a periodic point of $g$ of period $n k$ or less. However, $f$ must be $\Omega$-conjugate to a Kupka-Smale approximation (see [3]) which has only a finite number of periodic points of period $n k$ or less. Thus we have contradicted the assumption that $g$ is $\Omega$-conjugate to $f$. Q.E.D.

2. The stable manifolds of $\Omega$-stable diffeomorphisms. We now prove a "flattening lemma" which says that we can with an arbitrarily small perturbation of an embedding change it so there will be a flat spot around a point.

(2.1) LEMMA. Suppose $W$ is a submanifold of $R^{n}, w \in W, U$ is a neighborhood of $w$ in $R^{n}$, and $U^{\prime}$ is a compact neighborhood of w contained in $U$. Suppose further $h: U \rightarrow R^{n}$ is an embedding such that $h(w)=0$. Let $V$ be the tangent space to $h(W)$ at 0 and let $\pi: R^{n} \rightarrow V$ be orthogonal projection. Then given any $\varepsilon>0$, there exists a map $h^{\prime}: U \rightarrow R^{n}$ such that

(1) There exists a neighborhood $N$ of $w$ in $W$ such that $h^{\prime}(x)=\pi \cdot h(x)$ for $x \in N$.

(2) $h^{\prime}$ is $\varepsilon$ close to $h$ in the $C^{1}$ topology.

(3) $h(x)=h^{\prime}(x)$ for $x \notin U^{\prime}$.

Proof. Let $B(\delta)=\left\{v+e \mid v \in V, e \in V^{\perp}\right.$ and $\|v\| \leqq \delta$, $\left.\|e\| \leqq \delta\right\}$, and for $v \in V$ let $B_{v}(\delta)=\pi^{-1}(v) \cap B(\delta)$. Since $h(W)$ is tangent to $V$ at 0 there exists $\delta_{1}>0$ such that, for each $v \in V$ with $\|v\|<\delta_{1}, B_{v}\left(\delta_{1}\right) \cap h(W)$ is a single point. Define $u: B\left(\delta_{1}\right) \rightarrow$ $R^{n}$ by $u(x)=B_{\pi(x)}\left(\delta_{1}\right) \cap h(W)$. $u$ is simply projection on $h(W)$ along the planes orthogonal to $V$. Since the manifolds $h(W)$ and $V$ are tangent at 0 , the functions $\pi$ and $u$ are tangent at 0 , i.e.

$$
\lim _{\|x\| \rightarrow 0} \frac{\|\pi(x)-u(x)\|}{\|x\|}=0 .
$$

Hence we can choose $\delta_{2}<\delta_{1}$ such that

(1) $\delta_{2}<\varepsilon$ and $h^{-1}\left(B\left(\delta_{2}\right)\right) \subset U^{\prime}$.

(2) $\|\pi(x)-u(x)\|<(\varepsilon / 4 K)\|x\|$ when $\|x\|<\delta_{2}$ where $K=\sup \left\|d h_{z}\right\|$ over $z \in U^{\prime}$.

(3) $\left\|d \pi_{x}-d u_{x}\right\|<\varepsilon / 2 K$ when $\|x\|<\delta_{2}$.

We also choose a $C^{\infty}$ function $\sigma: R \rightarrow R$ such that $0 \leqq \sigma(t) \leqq 1, \sigma(t)=0$ when $|t|>\delta_{2}, \sigma(t)=1$ when $|t|<\delta_{2} / 4$, and $|d \sigma / d t|<2 / \delta_{2}$ for all $t$. Let $\rho(x)=\sigma(\|x\|)$.

Now define $h^{\prime}: U \rightarrow R^{n}$ by $h^{\prime}(z)=h(z)$ if $z \notin h^{-1}\left(B\left(\delta_{2}\right)\right)$ and $h^{\prime}(z)=h(z)+$ $\rho(h(z))(\pi(h(z))-u(h(z)))$ otherwise.

If $N$ is any neighborhood of $w$ in $W$ such that $N \subset h^{-1}\left(B\left(\delta_{2} / 4\right)\right)$ then for $z \in N$,

$$
\begin{aligned}
h^{\prime}(z) & =h(z)+\rho(h(z))(\pi(h(z))-u(h(z))) \\
& =h(z)+\pi(h(z))-h(z)=\pi(h(z)) .
\end{aligned}
$$

Also if $h(z) \in B\left(\delta_{2}\right)$,

and

$$
\left\|h(z)-h^{\prime}(z)\right\| \leqq \rho(h(z))\|\pi(h(z))-u(h(z))\|<\delta_{2}<\varepsilon,
$$

$$
\begin{aligned}
\left\|d h_{z}^{\prime}-d h_{z}\right\| & \leqq \rho(h(z))\left\|d \pi_{h(z)} \cdot d h_{z}-d u_{h(z)} \cdot d h_{z}\right\|+\left\|d \rho_{h(z)} \cdot d h_{z}\right\| \cdot\|\pi(h(z))-u(h(z))\| \\
& \leqq K(\varepsilon / 2 K)+\left(2 / \delta_{2}\right) K(\varepsilon / 4 K)\|h(z)\|<\varepsilon / 2+\varepsilon / 2=\varepsilon .
\end{aligned}
$$


(2.2) Lemma. If $f: M \rightarrow M$ is $\Omega$-stable and $p$ and $q$ are periodic points with $\operatorname{orb}(p)$ and orb $(q)$ attached then $\operatorname{dim} W^{s}(p)=\operatorname{dim} W^{s}(q)$.

Proof. We can assume without loss of generality that $W^{s}(p) \cap W^{u}(q) \neq \varnothing$ and $W^{u}(p) \cap W^{s}\left(q^{\prime}\right) \neq \varnothing$ where $q^{\prime} \in \operatorname{orb}(q)$. Suppose $\operatorname{dim} W^{s}(p)<\operatorname{dim} W^{s}(q)$ and let $x \in W^{s}(p) \cap W^{u}(q)$. We can perturb $f$ slightly to $f_{1}$, leaving it fixed on a neighborhood of orb $(x)=\left\{f^{n}(x) \mid-\infty<n<\infty\right\}$, so that $W^{s}\left(q^{\prime} ; f_{1}\right)$ and $W^{u}\left(p ; f_{1}\right)$ will have a point of transversal intersection. By making the perturbation $f_{1}$ close enough to $f$ we can guarantee that $f_{1}$ is $\Omega$-stable. We now have $x \in W^{s}\left(p, f_{1}\right) \cap W^{u}\left(q, f_{1}\right)$ and $W^{s}\left(q^{\prime} ; f_{1}\right)$ and $W^{u}\left(p ; f_{1}\right)$ have a point of transversal intersection. From this it follows easily that $x \in \Omega\left(f_{1}\right)$ (see [1]). We now choose a Kupka-Smale approximation (see [3] for definition) to $f_{1}, f_{2}$ which is sufficiently close to $f_{1}$ that there is an $\Omega$-conjugacy $h^{\prime}$ between $f_{1}$ and $f_{2}$ and that $\operatorname{dim} W^{s}\left(h^{\prime}(p)\right)=\operatorname{dim} W^{s}(p)$ and $\operatorname{dim} W^{u}\left(h^{\prime}(q)\right)=\operatorname{dim} W^{u}(q)$. Since $\operatorname{dim} W^{s}\left(h^{\prime}(p)\right)+\operatorname{dim} W^{u}\left(h^{\prime}(q)\right)<\operatorname{dim} M$ and these two manifolds have transverse intersection ( $f_{2}$ is Kupka-Smale) we have $W^{s}\left(\right.$ orb $\left.\left(h^{\prime}(p)\right)\right) \cap W^{u}\left(\right.$ orb $\left.\left(h^{\prime}(q)\right)\right)=\varnothing$.

Thus $f_{1}$ and $f_{2}$ cannot possibly be $\Omega$-conjugate, since any conjugacy would have to carry $x$ to a point in $W^{s}\left(h^{\prime}(p)\right) \cap W^{u}\left(h^{\prime}(q)\right)$. We have contradicted the assumption that $\operatorname{dim} W^{s}(p)<\operatorname{dim} W^{s}(q)$. A similar argument contradicts the reverse inequality and we conclude $\operatorname{dim} W^{s}(p)=\operatorname{dim} W^{s}(q)$. Q.E.D.

(2.3) LEMMA. If $f: M \rightarrow M$ is $\Omega$-stable and $p$ and $q$ are periodic points with orb $(p)$ attached to orb $(q)$ then $W^{s}($ orb $(p))$ and $W^{u}($ orb $(q))$ always intersect transversely.

Proof. Suppose to the contrary that $x$ is a point of nontransversal intersection of $W^{s}(p)$ and $W^{u}(q)$. By (2.2), $\operatorname{dim} W^{u}(p)+\operatorname{dim} W^{s}(q)=n$ so we can perturb $f$ to $f_{1}$ so that $f_{1}$ is $\Omega$-stable, so $f=f_{1}$ on a neighborhood of orb $(x)=\left\{f^{n}(x) \mid-\infty<n<\infty\right\}$, and so that $W^{u}\left(p ; f_{1}\right)$ and $W^{s}\left(q^{\prime} ; f_{1}\right)$ have a point of transversal intersection for some $q^{\prime} \in$ orb $(q)$. There exists an $\varepsilon^{\prime}>0$ such that if $g$ is $\varepsilon^{\prime}$ close to $f_{1}$ then $g$ is $\Omega$ conjugate to $f_{1}$ and the stable and unstable manifolds of $g$ corresponding to $W^{s}\left(q^{\prime} ; f_{1}\right)$ and $W^{u}\left(p ; f_{1}\right)$ respectively still have a point of transversal intersection.

$x$ is a point of nontransversal intersection of $W^{s}\left(p ; f_{1}\right)$ and $W^{u}\left(q ; f_{1}\right)$. Let $U$ be a neighborhood of $x$ in $M$ such that $U, f_{1}(U)$ and $f_{1}^{-1}(U)$ are disjoint and $\exp _{x}^{-1}$ is a diffeomorphism of $U$ onto its image in $T M_{x}$. Moreover we require that $U$ be sufficiently small that if $N_{s}=$ the component of $x$ in $W^{s}(p) \cap U$ and $N_{u}=$ the component of $x$ in $W^{u}(q) \cap U$ then $\bigcup_{i=1}^{\infty} f_{1}^{i}\left(N_{s}\right)$ and $\bigcup_{i=1}^{\infty} f_{1}^{-i}\left(N_{u}\right)$ are disjoint from $U$.

Let $V^{s}=T W^{s}(p)_{x}$ and let $V^{u}=T W^{u}(q)_{x}$, then there exists a nonzero vector $v \in V^{s} \cap V^{u}$. Choose $\delta>0$ such that $\exp _{x}(t v) \in U$ for $-\delta \leqq t \leqq \delta$ and let $J=$ $\exp _{x}(\{t v \mid-\delta \leqq t \leqq \delta\})$.

Choose $\varepsilon>0$ such that $\varepsilon<\varepsilon^{\prime}$ and if $g$ is $\varepsilon$ close to $f_{1}$ then $g$ is $\Omega$-conjugate to $f_{1}$. Let $k: f_{1}^{-1}(U) \rightarrow T M_{x}$ be given by $k=\exp _{x}^{-1} \cdot f_{1}$. Choose $\varepsilon_{1}>0$ so that if $k^{\prime}: f_{1}^{-1}(U)$ $\rightarrow T M_{x}$ is $\varepsilon_{1}$ close to $k$ then $\exp _{x} \cdot k^{\prime}$ is $\varepsilon / 4$ close to $f_{1}$. Now by (2.1) we can find 
$k_{1}: f_{1}^{-1}(U) \rightarrow T M_{x}$ such that (1) $k_{1}=k$ outside some neighborhood of $f_{1}^{-1}(x)$ which is contained in $f_{1}^{-1}(U),(2) k_{1}$ is $\varepsilon_{1}$ close to $k$, and (3) $k_{1}\left(W^{u}(q) \cap f_{1}^{-1}(U)\right)$ contains a neighborhood of 0 in $V^{u}$. Thus if we define $f_{2}: M \rightarrow M$ by $f_{2}(x)=f_{1}(x)$ for $x \notin f_{1}^{-1}(U)$ and $f_{2}(x)=\exp _{x} \cdot k_{1}$ for $x \in f_{1}^{-1}(U)$ then $f_{2}$ is $\varepsilon / 4$ close to $f_{1}$ and $W^{u}\left(q, f_{2}\right)$ contains a subinterval of $J$.

Similarly we can construct $f_{3}$ such that $f_{3}(x)=f_{2}(x)$ for $x \notin f_{1}(U), f_{3}$ is $\varepsilon / 4$ close to $f_{2}$ and $W^{s}\left(p, f_{3}\right)$ contains a subinterval of $J$. If $J^{\prime}$ is the subinterval of $J$ contained in $W^{u}\left(q_{1} f_{2}\right)$ then $f_{3}^{-i}\left(J^{\prime}\right)$ is disjoint from $f_{1}(U)$ so $f_{3}^{-i}\left(J^{\prime}\right)=f_{2}^{-i}\left(J^{\prime}\right)$ hence $J^{\prime} \subset W^{u}\left(q ; f_{3}\right)$.

Thus there is a neighborhood of $x$ in $J$ which is contained in $W^{s}\left(p ; f_{3}\right) \cap$ $W^{u}\left(q ; f_{3}\right)$. Also since $W^{u}\left(p ; f_{3}\right)$ and $W^{u}\left(q^{\prime} ; f_{3}\right)$ have a point of transversal intersection each point of $W^{s}\left(p ; f_{3}\right) \cap W^{u}\left(q ; f_{3}\right)$ is nonwandering (see the theorem of $\S 1$ in [1]).

This leads to a contradiction however since $f_{3}$ is $\Omega$-conjugate to $f_{1}$ which is $\Omega$ stable and hence $f_{3}$ is $\Omega$-conjugate to a sufficiently close Kupka-Smale approximation to $f_{1}$. However, any Kupka-Smale approximation has only a countable number of points in the intersection of a stable and unstable manifold of complimentary dimensions, while $\operatorname{dim} W^{s}\left(p ; f_{3}\right)+\operatorname{dim} W^{u}\left(q ; f_{3}\right)=n$ and their intersection contains an interval of nonwandering points. Hence our initial assumption of the existence of a nontransversal point of intersection must have been false. Q.E.D.

Proof of Theorem 2. Choose $\varepsilon>0$ so that if $g$ is $\varepsilon$ close to $f$ in the $C^{1}$ metric then $g$ is $\Omega$-stable and $\Omega$-conjugate to $f$. By hypothesis there are periodic points $p_{1}$, $q_{1} \in \Lambda_{1}$ and $p_{2}, q_{2} \in \Lambda_{2}$ such that $W^{u}\left(q_{1}\right) \cap W^{s}\left(q_{2}\right) \neq \varnothing$ and $W^{s}\left(p_{1}\right) \cap W^{u}\left(p_{2}\right) \neq \varnothing$. Suppose without loss of generality that $\operatorname{dim} W^{s}\left(p_{1}\right)+\operatorname{dim} W^{u}\left(p_{2}\right) \geqq n$ (otherwise consider $q_{1}$ and $\left.q_{2}\right)$. Let $w \in W^{u}\left(q_{1}\right) \cap W^{s}\left(q_{2}\right)$ then we can obtain an approximation $f_{1}$ which is $\varepsilon / 4$ close to $f$ in the $C^{1}$ metric, which is equal to $f$ on the closure of orb $(w) \cup \Lambda_{1} \cup \Lambda_{2}$, and which has the property that $W^{s}\left(p_{1} ; f_{1}\right)$ and $W^{u}\left(p_{2} ; f_{1}\right)$ have a point of transversal intersection.

The argument of (7.6) (a) in Smale's paper [3] shows that $W^{s}\left(\right.$ orb $\left.\left(q_{1}\right) ; f_{1}\right)$ is dense in $\Lambda_{1}$ and $W^{u}$ (orb $\left.\left(q_{2}\right) ; f_{1}\right)$ is dense in $\Lambda_{2}$. Hence $W^{s}\left(\right.$ orb $\left.\left(q_{1}\right) ; f_{1}\right)$ is dense in $W^{s}\left(\Lambda_{1} ; f_{1}\right)$ and $W^{u}\left(\right.$ orb $\left.\left(q_{2}\right) ; f_{1}\right)$ is dense in $W^{u}\left(\Lambda_{2} ; f_{1}\right)$. By the continuity of local stable manifolds (see 3.2 of [2]) there must exist a point of intersection of $W^{s}\left(\operatorname{orb}\left(q_{1}\right) ; f_{1}\right)$ and $W^{u}\left(\operatorname{orb}\left(q_{2}\right) ; f_{1}\right)$ since $W^{s}\left(p_{1} ; f_{1}\right)$ and $W^{u}\left(p_{2} ; f_{1}\right)$ intersect transversely. That is, considering the diffeomorphism $f_{1}$, orb $\left(q_{1}\right)$ and orb $\left(q_{2}\right)$ are attached. Hence by $(2.2), \operatorname{dim} W^{s}\left(q_{1}\right)=\operatorname{dim} W^{s}\left(q_{2}\right)$. Thus $\operatorname{dim} E^{s}\left(\Lambda_{1}\right)=\operatorname{dim} E^{s}\left(\Lambda_{2}\right)$.

We now assume that there is no lower bound on the angles of intersection of $W^{s}\left(\Lambda_{1}\right)$ and $W^{u}\left(\Lambda_{2}\right)$ and derive a contradiction to $\Omega$-stability of $f$.

We first choose $\beta>0$ such that

(1) If we have unit vectors $v_{1}, v_{2} \in T M_{x}$ with the angle between $v_{1}$ and $v_{2}$ less than $\beta$ then there exists $L: T M_{x} \rightarrow T M_{x}$ such that $L\left(v_{1}\right)=v_{2}$ and

$$
\|L-\mathrm{id}\|<(\varepsilon / 20)\left(\sup \left\|d f_{x}\right\|+\varepsilon\right)^{-1} \text {. }
$$

(2) For any $x \in \Lambda_{1}$ or $\Lambda_{2}$ the angle between $E_{x}^{s}$ and $E_{x}^{u}$ is greater than $\beta$. 
Suppose there are points $x_{1} \in \Lambda_{1}, x_{2} \in \Lambda_{2}$ and $z$ such that $W^{s}\left(x_{1} ; f_{1}\right)$ and $W^{u}\left(x_{2} ; f_{1}\right)$ intersect at $z$ with the angle between them less than $\beta / 2$ (this implies $\left.z \notin \Lambda_{1} \cup \Lambda_{2}\right)$. If $W^{s}\left(x_{1} ; f_{1}\right)$ does not intersect $W^{u}\left(x_{2} ; f_{1}\right)$ transversely then we approximate $f_{1}$ by $f_{2}$ so that $f_{2}$ is $\varepsilon / 4$ close to $f_{1}$ in the $C^{1}$ metric, $f_{2}$ equals $f_{1}$ on a neighborhood of $\Lambda_{1} \cup \Lambda_{2} \cup$ orb $(w)$ and $W^{s}\left(x_{1} ; f_{2}\right)$ intersects $W^{u}\left(x_{2} ; f_{2}\right)$ transversely at $z$ but with the angle between them less than $\beta$.

As before $W^{s}\left(\right.$ orb $\left.\left(q_{1}\right) ; f_{2}\right)$ is dense in $\Lambda_{1}$ and $W^{u}\left(\right.$ orb $\left.\left(q_{2}\right) ; f_{2}\right)$ is dense in $\Lambda_{2}$. Hence we can conclude from the continuity of the local stable manifolds (3.2 of [2]) that there is a point $z^{\prime}$ near $z$ where $W^{s}\left(\right.$ orb $\left.\left(q_{1}\right) ; f_{2}\right)$ intersects $W^{u}\left(\operatorname{orb}\left(q_{2}\right) ; f_{2}\right)$ and the angle between them is less than $\beta$. Hence there are unit vectors $v^{s} \in T M_{z^{\prime}}$ tangent to $W^{s}\left(\operatorname{orb}\left(q_{1}\right) ; f_{2}\right)$ and $v^{u} \in T M_{z^{\prime}}$ tangent to $W^{u}\left(\operatorname{orb}\left(q_{2}\right) ; f_{2}\right)$ and there is an isomorphism $L: T M_{z^{\prime}} \rightarrow T M_{z^{\prime}}$ such that $L\left(v^{u}\right)=v^{s}$ and $\left\|L \cdot d f_{2}-d f_{2}\right\|<\varepsilon / 20$.

Let $y^{\prime}=f_{2}^{-1}\left(z^{\prime}\right)$ and define $G: T M_{y} \rightarrow T M_{z^{\prime}}$ by $G=L \cdot d f_{2}$. Let $Q=\left\{f_{2}^{n}\left(y^{\prime}\right) \mid n \neq 0\right\}$ $\cup \operatorname{orb}(w)$. We now apply (1.1) to the diffeomorphism $f_{2}$ with $\theta=y^{\prime}, G$ as we have constructed it and $R$ a compact neighborhood $\Lambda_{1} \cup \Lambda_{2} \cup Q$ which is disjoint from $y^{\prime}$.

We obtain a diffeomorphism $g: M \rightarrow M$ which is $\Omega$-stable and $\varepsilon$-close to $f$ in the $C^{1}$ metric. Since $g=f_{2}$ on $R$ we have $d g^{n}\left(v^{s}\right)=d f_{2}^{n}\left(v^{s}\right)$ for $n \geqq 0$. On the other hand $d g^{-n}\left(v^{s}\right)=d f_{2}^{-n}\left(v^{u}\right)$ for $n>0$. So $v^{s}$ is tangent to both $W^{s}\left(\operatorname{orb}\left(q_{1}\right) ; g\right)$ and $W^{u}$ (orb $\left.\left(q_{2}\right) ; g\right)$. Hence by $(2.3) g$ cannot be $\Omega$-stable which is a contradiction. Q.E.D.

\section{REFERENCES}

1. R. Abraham and S. Smale, Non-genericity of $\Omega$-stability, Proc. Sympos. Pure Math., vol. 14, Amer. Math. Soc., Providence, R. I., 1970, pp. 5-8.

2. M. W. Hirsch and C. Pugh, Stable manifolds and hyperbolic sets, Proc. Sympos. Pure Math., vol. 14, Amer. Math. Soc., Providence, R. I., 1970, pp. 133-163.

3. S. Smale, Differentiable dynamical systems, Bull. Amer. Math. Soc. 73 (1967), 747-817. MR 37 \#3598.

4. —, The $\Omega$-stability theorem, Proc. Sympos. Pure Math., vol. 14, Amer. Math. Soc., Providence, R. I., 1970, pp. 289-297.

Massachusetts Institute of Technology, CAMBridge, MassachusetTs 02139

NORTHWESTERN UNIVERSITY, EVANSTON, ILLINOIS 60201 